\title{
'Failure' of laser prophylaxis in an eye with stickler syndrome
}

\begin{abstract}
Background: Stickler syndrome is one of the most common inherited connective tissue disorders and is an important cause of pediatric vision loss due to a high risk of retinal detachment in these patients.

Methods: Case report.

Case summary: This case reports describes the clinical course of a 10 year old boy with Sticklers Syndrome who underwent bilateral peripheral laser prophylaxis. During routine follow up, he was found to have an asymptomatic giant retinal tear (GRT) with limited sub-retinal fluid expansion due to prior prophylactic laser. He underwent surgery with vitrectomy and scleral buckle with vision remaining at 20/25 at 6 month follow up.

Conclusion: Although the utility of laser prophylaxis in Stickler patients is debated, this case demonstrates that after laser prophylaxis, even if GRT develops, expansion can be limited. Laser prophylaxis along with frequent examinations, can prevent development of PVR and complex detachments and preserve macular function with excellent visual outcome.
\end{abstract}

Keywords: stickler syndrome, retinal detachment, laser prophylaxis, giant retinal tear
Volume II Issue 4 - 202 I

\author{
Mateo A Blair,' David T Dao, ${ }^{3}$ Michael P \\ Blair, ${ }^{1,3}$ Michael J Shapiro ${ }^{1,2}$ \\ 'Retina Consultants Ltd., Des Plaines, USA \\ 2Department of Ophthalmology and Visual Science, University \\ of Illinois, USA \\ ${ }^{3}$ Department of Ophthalmology and Visual Science, University \\ of Chicago, USA
}

Correspondence: Michael P Blair, Retina Consultants Ltd. 2454 E. Dempster St., Suite 400, Des Plaines, Illinois, USA, Email michaelpaulblair@gmail.com

Received: June 27, 2021 | Published: July 28, 2021

\section{Introduction}

Type 1 Sticklersyndrome is caused by mutation in the COL2A1 gene and is characterized by orofacial abnormalities, early arthropathy, and abnormal vitreous. ${ }^{1}$ Stickler syndrome is one of the most common inherited connective tissue disorders and is an important cause of pediatric vision loss due to a high risk of retinal detachment in these patients. ${ }^{2}$ Retinal detachment in Stickler syndrome is commonly associated with giant retinal tears (GRT) with a high rate of proliferative vitreoretinopathy (PVR) limiting the success rate of surgery. ${ }^{3}$ Retrospective studies have suggested that prophylaxis of retinal detachment with laser or cryotherapy can reduce the risk of retinal detachment. ${ }^{4}$ However, periodic routine monitoring is critical as retinal tears may still develop after prophylaxis. In this case report, we describe the clinical and surgical course of a giant retinal tear developing after bilateral laser prophylaxis.

\section{Case report}

An asymptomatic 10-year-old boy with bilateral hearing loss, whose father and two sisters were known to have Stickler Syndrome type 1, presented for fundus examination. His Snellen acuity was $20 / 25$ OU and perivascular lattice was present. Genetic testing confirmed an autosomal dominant heterozygous mutation of c. $2818 \mathrm{C}>\mathrm{T}$ in COL2A1. He underwent bilateral peripheral laser prophylaxis, treating from ora serrata to equator to limit giant retina tear (GRT) development and/or expansion and thus reduce the chance of retinal detachment (RD), proliferative vitreoretinopathy (PVR) and visual loss. Four years later, during a routine examination, at which time he was asymptomatic, posterior vitreous detachment and GRT were noted. Examination under anaesthesia 2 weeks later, showed progression of fluid into but not past laser prophylaxis. Scleral buckle and vitrectomy were performed. A 6 month follow up, his Snellen acuity was $20 / 25$ OU with mild cataract, no evidence of glaucoma and the retina was completely attached.

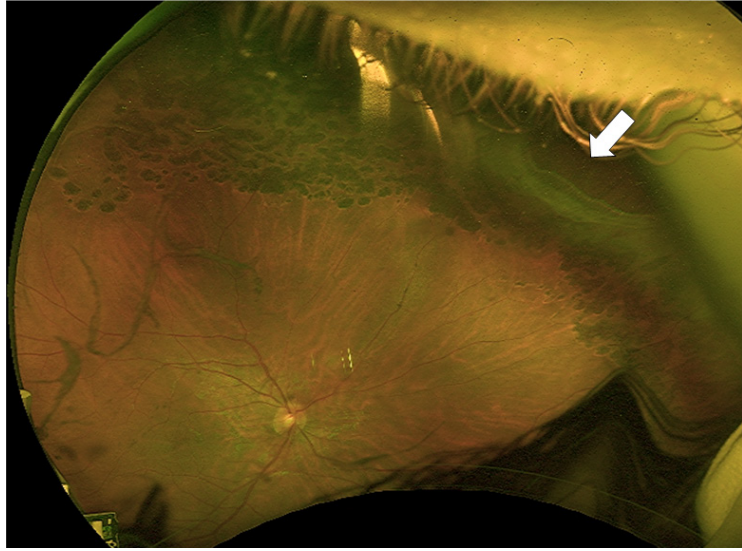

Figure I Optos wide field fundus photo of an asymptomatic giant retinal tear (white arrow) of the right eye with limitation of subretinal fluid expansion by prophylactic laser in a I0-year-old boy with Stickler syndrome.

\section{Discussion}

Stickler syndrome is a one of the most frequently inherited connective tissue disorders and a frequent cause of pediatric blindness due to the high incidence of retinal detachment. Early diagnosis of retinal tear or detachment is challenging due to the difficulty of examining pediatric patients. While prophylaxis of retinal detachment with laser or cryotherapy has been explored, there is controversy regarding the safety and effectiveness of this approach with no clear consensus..$^{5-7}$ The most standardized approach to prophylaxis was presented by the Cambridge group case-control study which demonstrated a significant reduction in unfavorable outcomes with a single row of cryotherapy prophylaxis at the ora serrata. ${ }^{4}$ However, it is important to note that prophylaxis does not replace the need for regular periodic examination because, as our case demonstrates, 
asymptomatic GRT can still occur. ${ }^{2}$ Without treatment GRT can lead to PVR and complex retinal detachment configuration which makes surgical repair more technically challenging and visual outcome less certain. In our case, although laser prophylaxis failed to prevent retinal detachment, it did limit GRT expansion and together with frequent examination, prevented PVR and led to an excellent visual outcome after surgical repair.

\section{Conclusions}

In this case report, we demonstrate the clinical course of a giant retinal tear in a young patient with Stickler syndrome who received laser prophylaxis. Although the utility of laser prophylaxis in Stickler patients is debated, this case demonstrates that even if laser prophylaxis fails to prevent retinal detachment, if combined with a program of periodic examination, expansion can be limited. This can prevent development of PVR and complex detachments, preserve macular function and maintain excellent visual outcome. Future randomized controlled studies are needed to determine clear recommendations for prevention of retinal detachment in patients with Stickler syndrome.

\section{Patient consent}

Consent to publish the case report was not obtained. This report does not contain any personal information that could lead to the identification of the patient.

\section{Funding}

No funding or grant support.

\section{Authorship}

All authors attest that they meet the current ICMJE criteria for authorship.

\section{Conflicts of interest}

All authors have no financial disclosures.

\section{Acknowledgments}

None.

\section{References}

1. Stickler GB, W Hughes, P Houchin. 2001. Clinical Features of Hereditary Progressive Arthro-Ophthalmopathy (Stickler Syndrome): A Survey. Genet Med. 2001;3(3):192-196.

2. Shapiro, Michael J, Michael P Blair, et al. 2018. The Importance of Early Diagnosis of Stickler Syndrome: Finding Opportunities for Preventing Blindness. Taiwan J Ophthalmol. 2018;8(4):189-195.

3. Abeysiri, Poorna, Catey Bunce, et al. Outcomes of Surgery for Retinal Detachment in Patients with Stickler Syndrome: A Comparison of Two Sequential 20-Year Cohorts. Graefes Arch Clin Exp Ophthalmol. 2007;245(11):1633-1638.

4. Fincham, Gregory S, Laura Pasea, et al. Prevention of Retinal Detachment in Stickler Syndrome: The Cambridge Prophylactic Cryotherapy Protocol. Ophthalmology. 2014;121(8):1588-1597.

5. Coussa, Razek Georges, Jonathan Sears, et al. Stickler Syndrome: Exploring Prophylaxis for Retinal Detachment. Current Opinion in Ophthalmology. 2019;30(5):306-313.

6. Boysen, Kirstine B, Morten La Cour, et al. Ocular Complications and Prophylactic Strategies in Stickler Syndrome: A Systematic Literature Review. Ophthalmic Genetics. 2020;41(3):223-234.

7. Morris, Robert E, Edward Scott Parma, Nathaniel H. Robin, et al. Stickler Syndrome (SS): Laser Prophylaxis for Retinal Detachment (Modified Ora Secunda Cerclage, OSC/SS). Clinical Ophthalmol. 2021;15:19-29. 